\title{
Thanks to reviewers
}

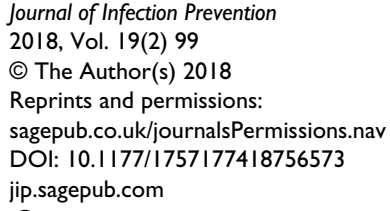

Journal of Infection Prevention 2018, Vol. 19(2) 99

(C) The Author(s) 2018

Reprints and permissions:

sagepub.co.uk/journalsPermissions.nav

DOI: | 0.1 |77/|757|774|8756573

jip.sagepub.com

(S)AGE

The journal sincerely thanks the following individuals who reviewed one or more manuscripts during 2017:

Adams, Debra

Alqumber, Mohammed

Bak, Aggie

Baxter, Elaine

Beck, Charles

Beckett, Gail

Blenkharn, Ian

Bradley, Craig

Brannigan, Eimear

Burnett, Emma

Carter, Yvonne

Chandra, Mudit

Child, Jenny

Cochrane, Joan

Cole, Mark

Cooper, Mike

Cooper, Tracey

Curran, Evonne

Davies, Joanna

Denton, Andrea

Dunnett, Andrew

Dyson, Judith

Elgohari, Suzanne

Elliott, Paul

Falconer, Michelle

Flynn, Julie

Foster, Dona

Fry, Carole

Garvey, Mark

Gosadi, Ibrahim

Grainger, Carrie

Grimmond, Terry

Hammer, David

Hodgson, Gillian

Hoffman, Peter

Humphreys, Paul

Jeanes, Annette

Jenkins, Lynda
Jones, Barry

Jones, Graeme

Kermode, Margaret

Kiernan, Martin

Kilpatrick, Claire

Loveday, Heather

MacDonald, Jennifer

MacDonald, Pauline

Mackay, Ian

Magill-Cuerden, Julia

McCulloch, Janet

Myron, Rowan

Nagar, Aaron

Nevill, Michael

Overton, Charlotte

Prieto, Jacqui

Reilly, Jacqui

Ritchie, Lisa

Robinson, Jude

Ross, Elaine

Saeed, Kordo

Serou, Naresh

Shepherd, Lesley

Smales, Caroline

Smith, Andrew

Spencer, Fay

Storr, Julie

Thomlinson, Diane

Tudor, Terry

van de Mortel, Thea

Weaving, Paul

Westbury, Jan

Weston, Debbie

Whitehouse, Tony

Wigglesworth, Neil

Williams, Dafydd

Wilson, Peter

Xuereb, Debbie 